\title{
Editorial: Data-Driven Integrated Computational Materials Engineering for High-Entropy Materials
}

\author{
William Yi Wang ${ }^{1 *}$, Yong Zhang ${ }^{2 *}$ and Peter K. Liaw ${ }^{3 *}$ \\ ${ }^{1}$ State Key Laboratory of Solidification Processing, Northwestern Polytechnical University, Xían, China, ${ }^{2}$ Beijing Advanced \\ Innovation Center of Materials Genome Engineering, State Key Laboratory for Advanced Metals and Materials, University of \\ Science and Technology Beijing, Beijing, China, ${ }^{3}$ Department of Materials Science and Engineering, The University of Tennessee, \\ Knoxville, TN, United States
}

Keywords: high-entropy materials, high-throughput calculations, data-driven modeling, machine learning, empirical models

Editorial on the Research Topic

Data-Driven Integrated Computational Materials Engineering for High-entropy Materials

High-entropy materials (HEMs), including alloys, ceramics, oxides, and semiconductors, have attracted enormous activities to investigate their attractive/excellent properties and potential critical applications (Zhang et al., 2014; Santodonato et al., 2015; Wang et al., 2017; Zhang et al., 2017; Ding et al., 2019; Qin et al., 2019; Shi et al., 2019; Li et al., 2020; Oses et al., 2020; Wright and Luo, 2020). With the contributions of multiple solute atoms, higher configurational entropy is expected, driving a tendency to form simple solid solutions (amorphous or crystalline) rather than complex microstructures with many compounds. Together with the high-throughput experiments, the Integrated Computational Materials Engineering (ICME) approach consisting of calculation of phase diagrams (CALPHAD), ab initio molecular dynamics, phase-field simulations, finite element calculations, and Monte Carlo has been supported by the Material Genome Initiative/Engineering (MGI/MGE) and is boosting the database (Liu et al., 2040; The Minerals Metals Materials Society, 2015; Wang et al., 2019; Wang et al., 2020). Toward the inheritable integrated intelligent manufacturing era, the data-driven ICME is critical to accelerate the discoveries and applications of novel advanced HEMs.

The frontiers in HEMs has been reviewed and highlighted, presenting the recent research on the fundamental understanding and theoretical modeling of the composition-processingmicrostructures-properties-performance relationship of HEMs, possessing tailorable properties that far surpass their conventional alloys, such as high strength, ductility, ultrahigh melting, electrical and thermal conductivities, corrosion resistance, oxidation resistance, fatigue, and wear resistance. These properties will undoubtedly make HEMs of interest for use in biomedical, structural, mechanical, and energy applications. With the novel and exciting nature of HEMs, they are poised for significant growth and present a perfect opportunity for a new symposium and research field.

In this special issue, five manuscripts are combined here, discussing several key aspects related to the novel HEMs. In the review article entitled "High-Throughput Calculations for High-Entropy Alloys: A Brief Review," Li et al. highlighted four different computational methods commonly utilized in the development of HEM compositions, including empirical models, first-principle calculations, CALPHAD, and machine learning. The empirical model and the machine learning are both based on summary and analysis, while the latter is more believable for the use of multiple algorithms. The first-principle calculations are based on quantum mechanics and several open-source databases, and they also provide the finer atomic information for the thermodynamic analysis of CALPHAD and 
machine learning. It illustrates the advantages, disadvantages, and application range of these techniques, and comparisons with each other to provide some guidance for the HEA study.

As for the multicomponent HEMs, it is essential to reveal the contributions of local chemical environments (i.e., the so-called microstates, clusters, short range orders, and so on) to their various chemical and physical properties. In the article named "Structural and Thermodynamic Properties of the High-Entropy Alloy AlCoCrFeNi based on First-Principles Calculations," Wu et al. combined structural modeling with the similar atomic environment (SAE) method and first-principle simulations with the modified mean-field potential approach to construct the supercells of AlCoCrFeNi. Based on the predicted lattice and magnetic structure as well as the thermodynamic properties, a strong local lattice distortion of $\mathrm{AlCoCrFeNi}$, compared with typical $3 \mathrm{~d}$ high-entropy alloys, is discussed. It is found that the equilibrium volumes, bulk modulus, and shock Hugoniot agree well with available experimental and theoretical results, demonstrating the validity and reliability of these methods in the study of dynamic properties of $\mathrm{AlCoCrFeNi}$, and providing a promising scheme for accessing the dynamic properties of sophisticated high-entropy alloys.

Beyond the solid-solution strengthening, the phase stability at the intermediate temperature and the effects of oxygen addition on the deformation behavior during tensile tests are needed to be well understood. In the research article entitled "Phase Stability and Deformation Behavior of TiZrHfNbO High-Entropy Alloys," Wu et al. investigated the formation of hexagonal close-packed (HCP) solid-solution precipitates enriched by $\mathrm{Hf}, \mathrm{Zr}$, and $\mathrm{O}$ atoms in the $(\mathrm{TiZrHfNb})_{100-\mathrm{x}} \mathrm{O}_{\mathrm{x}}$, in terms of $\mathrm{X}$-ray diffraction, transmission electron microscopy, and atom probe tomography. It is found that the doped oxygen increases the yield strength and maintains the ductility for these HEMs annealed at $1,273 \mathrm{~K}$, while the formation of HCP precipitates after annealing at $873 \mathrm{~K}$ deteriorates the plasticity significantly. To unveil the deformation behaviors, in situ synchrotron X-ray diffraction experiments are performed. The single-crystal elastic constants and shear elastic anisotropy of HEAs with and without oxygen are also calculated, which are similar to those of the "gum metal" Ti alloy.

In the review article entitled "Thermal-Mechanical Processing and Strengthen in AlxCoCrFeNi High-Entropy Alloys," $\mathrm{Li}$ et al. investigated the phase-transition

\section{REFERENCES}

Ding, Q., Zhang, Y., Chen, X., Fu, X., Chen, D., Chen, S., et al. (2019). Tuning element distribution, structure and properties by composition in high-entropy alloys. Nature 574, 223-227. doi:10.1038/s41586-019-1617-1

Li, R., Xie, L., Wang, W. Y., Liaw, P. K., and Zhang, Y. (2020). High-throughput calculations for high-entropy alloys: a brief review. Front. Mater. 7, 290. doi:10.3389/fmats.2020.00290

Liu, X., Furrer, D., Kosters, J., and Holmes, J. (2040). A roadmap for integrated, multiscale modeling and simulation of materials and systems. Washington, DC: NASA.

Oses, C., Toher, C., and Curtarolo, S. (2020). High-entropy ceramics. Nat. Rev. Mater. 5, 295-309. doi:10.1038/s41578-019-0170-8

Qin, G., Chen, R., Zheng, H., Fang, H., Wang, L., Su, Y., et al. (2019). Strengthening FCC-CoCrFeMnNi high entropy alloys by Mo addition. J. Mater. Sci. Technol. 35, 578-583. doi:10.1016/j.jmst.2018.10.009 mechanisms and mechanical properties of HEMs under the thermal-mechanical coupling effect. It explored the microstructural evolution, formation of secondary phases at high and/or intermediate temperatures, and their effect on mechanical properties of the classical AlxCoCrFeNi HEMs. Since an alloy with a relatively low stacking fault energy regulates the stacking fault energy, triggering the twin/transformation-induced plasticity effects, the stacking fault energy, and its local structural phase transformation are highlighted and recommended to be considered as criteria in optimizing the mechanical properties and deformation mechanisms of HEMs.

Motivated by the outstanding mechanical properties of binary and ternary carbides, San and Ching wrote a research article named "Subtle Variations of the Electronic Structure and Mechanical Properties of High Entropy Alloys With 50\% Carbon Composites." On the basis of systematic large-scale $a b$ initio simulations, subtle differences in their structures, and electronic and mechanical properties are revealed and discussed in detail. Fifteen supercell models with 512 atoms with an equal composition of $\mathrm{C}$ and HEAs occupying the sublattices of the rock-salt structure were constructed. Through utilizing the electronic structure, interatomic bonding, and partial charge distributions, fundamental understanding of these novel high-entropy carbides and their mechanical properties is provided. The corresponding methods mentioned here can also be extended in the development of advanced complex HEMs, including high-entropy oxides, nitrides, borides, and silicides. The large database can play an important and valuable role in the design and synthesis of high-entropy ceramics.

We would like to thank the supports from all the authors, reviewers, and editors for their dedication and responsibility. Those great contributions guarantee the publication of this excellent special issue in time and are appreciated.

\section{AUTHOR CONTRIBUTIONS}

WW, PL, and YZ edited, reviewed, and summarized the special issue. All authors contributed to the general discussion.

Santodonato, L. J., Zhang, Y., Feygenson, M., Parish, C. M., Gao, M. C., Weber, R. J., et al. (2015). Deviation from high-entropy configurations in the atomic distributions of a multi-principal-element alloy. Nat. Commun. 6, 5964. doi:10. 1038/ncomms6964

Shi, P., Ren, W., Zheng, T., Ren, Z., Hou, X., Peng, J., et al. (2019). Enhanced strength-ductility synergy in ultrafine-grained eutectic high-entropy alloys by inheriting microstructural lamellae. Nat. Commun. 10, 489. doi:10.1038/ s41467-019-08460-2

The Minerals Metals Materials Society (2015). Modeling across scales: a roadmapping study for connecting materials models and simulations across length and time scales. Warrendale, PA: TMS.

Wang, W. Y., Shang, S. L., Wang, Y., Han, F., Darling, K. A., Wu, Y., et al. (2017). Atomic and electronic basis for the serrations of refractory high-entropy alloys. Npj Comp. Mater. 3, 23. doi:10.1038/s41524-0170024-0 
Wang, W. Y., Li, J., Liu, W., and Liu, Z-K. (2019). Integrated computational materials engineering for advanced materials: a brief review. Comp. Mater. Sci. 158, 42-48. doi:10.1016/j.commatsci.2018. 11.001

Wang, W. Y., Tang, B., Lin, D., Zou, C., Zhang, Y., Shang, S.-L., et al. (2020). A brief review of data-driven ICME for intelligently discovering advanced structural metal materials: insight into atomic and electronic building blocks. J. Mater. Res. 35, 872-889. doi:10.1557/ jmr.2020.43

Wright, A. J., and Luo, J. (2020). A step forward from high-entropy ceramics to compositionally complex ceramics: a new perspective. J. Mater. Sci. 55, 9812-9827. doi:10.1007/s10853-020-04583-w

Zhang, Y., Zuo, T. T., Tang, Z., Gao, M. C., Dahmen, K. A., Liaw, P. K., et al. (2014). Microstructures and properties of high-entropy alloys. Prog. Mater. Sci. 61, 1-93. doi:10.1016/j.pmatsci.2013.10.001
Zhang, Y., Liu, J. P., Chen, S. Y., Xie, X., Liaw, P. K., Dahmen, K. A., et al. (2017). Serration and noise behaviors in materials. Prog. Mater. Sci. 90, 358-460. doi:10. 1016/j.pmatsci.2017.06.004

Conflict of Interest: The authors declare that the research was conducted in the absence of any commercial or financial relationships that could be construed as a potential conflict of interest.

Copyright (c) 2021 Wang, Zhang and Liaw. This is an open-access article distributed under the terms of the Creative Commons Attribution License (CC BY). The use, distribution or reproduction in other forums is permitted, provided the original author(s) and the copyright owner(s) are credited and that the original publication in this journal is cited, in accordance with accepted academic practice. No use, distribution or reproduction is permitted which does not comply with these terms. 